\title{
Tracheobronchial Tree
}

National Cancer Institute

\section{Source}

National Cancer Institute. Tracheobronchial Tree. NCI Thesaurus. Code C117875.

An anatomical structure comprising of trachea, bronchi, and bronchioles that terminate

with the alveolar ducts, sacs, and alveoli. 